\title{
DETECTION AND IDENTIFICATION OF LOSS OF EFFICIENCY FAULTS OF FLIGHT ACTUATORS
}

\author{
DANIEL OSSMANN $^{a, *}$, ANDREAS VARGA $^{a}$ \\ ${ }^{a}$ Institute of System Dynamics and Control \\ DLR Oberpfaffenhofen, D-82234 Wessling, Germany \\ e-mail: \{daniel.ossmann, andreas.varga\}@dlr.de
}

\begin{abstract}
We propose linear parameter-varying (LPV) model-based approaches to the synthesis of robust fault detection and diagnosis (FDD) systems for loss of efficiency (LOE) faults of flight actuators. The proposed methods are applicable to several types of parametric (or multiplicative) LOE faults such as actuator disconnection, surface damage, actuator power loss or stall loads. For the detection of these parametric faults, advanced LPV-model detection techniques are proposed, which implicitly provide fault identification information. Fast detection of intermittent stall loads (seen as nuisances, rather than faults) is important in enhancing the performance of various fault detection schemes dealing with large input signals. For this case, a dedicated fast identification algorithm is devised. The developed FDD systems are tested on a nonlinear actuator model which is implemented in a full nonlinear aircraft simulation model. This enables the validation of the FDD system's detection and identification characteristics under realistic conditions.
\end{abstract}

Keywords: aerospace engineering, fault detection and diagnosis, loss of efficiency type of faults, flight actuator faults.

\section{Introduction}

There are several classes of flight actuator faults whose early detection and timely handling contribute to safe operation of aircraft, avoid excessive fuel consumption (with all associated negative environmental effects) and increase the aircraft operational autonomy. Therefore, the deployment of fault detection and diagnosis (FDD) systems for monitoring and identification of these faults is of paramount importance for large civil transport aircraft. Once a certain type of fault has been identified, appropriate reconfiguration actions may take place in order to eliminate the effects of malfunctioning and to ensure acceptable performance. A standard certification requirement for aircraft is that no single fault can lead to catastrophic consequences. To fulfill this aim, the flight control system augmented with appropriate FDD systems must reliably operate over the whole flight envelope, for a large variety of maneuvers, in the presence of uncertainties such as external wind disturbances or variations of aircraft parameters.

Robust linear parameter-varying (LPV) model based fault diagnosis approaches for the main categories of

\footnotetext{
*Corresponding author
}

additive type flight actuator faults have been developed by Varga and Ossmann (2014) using a component level approach, where each flight actuator is independently monitored using exclusively locally available signal measurements. The main advantages of such an approach is that the fault isolation is implicitly provided by the fault detection scheme and the resulting fault detection filters are simpler to implement than filters resulting from system-wide approaches. Due to these advantages this approach is the current industrial state-of-the-practise. However, the development of similar component level methods for parametric or multiplicative types of faults, such as various loss of efficiency (LOE) faults, raises challenges because of the typical lack of a strong fault detectability property. In other words, parametric faults typically lead to changes in the actuator dynamics and therefore have asymptotically vanishing effects which need very short fault detection times. To fill this gap, in this paper we address the problem of detecting and identifying a parametric type of LOE faults by proposing a complete methodology for the design of a dedicated model based FDD system on the actuator level.

The detection and identification of the LOE type of flight actuator faults has been intensively studied (see, 
e.g., the works of Caglayan et al. (1988), Marton and Ossmann (2012), Eykeren et al. (2012) and the references cited therein). Early detection of these faults is important to prevent, via suitable control reconfiguration schemes, undesired effects on the flight mechanics, aircraft stability and fuel consumption. There exists LOE faults which, although parametric, can be still addressed using methods suited for additive faults. An example of such an LOE fault is an unmeasurable change in the actuator's input signal, caused, for example, by incorrect signal transmissions from the flight control computer to the actuator or induction of an undesired current by external effects. The result is a corrupted input-output transfer gain, as discussed by Boškovic et al. (2005). Suitable methods to monitor these faults are given by Varga and Ossmann (2014).

In this paper we focus on developing methods suitable for the detection and identification of parametric (or multiplicative) LOE faults, whose main effects are changes in the dynamical behavior of the actuator. Such LOE faults are caused by a leakage within the actuator, a damage in the control surface or even the disconnection of the actuator rod from the control surface, which represents a worst-case scenario of the LOE, as the efficiency of the control surface goes to zero. The disconnection of the actuator, as well as any damage of the control surface, leads to an increased bandwidth of the actuator due to the reduced aerodynamic loads transferred to the actuator piston. In contrast, due to a reduced available pressure, leakages cause a decrease in the bandwidth. For the development of a fault tolerant flight control system, robust detection and identification of such a parametric type of LOE faults over the whole flight envelope, for different pilot maneuvers and wind conditions, and over the whole range of uncertain parameter variations is an important prerequisite.

A special type of LOE discussed separately in this paper is the so-called stall load phenomenon. Stall loads are intermittent saturations of the actuator, which occur when the aerodynamics loads temporarily exceed the maximum available actuator force produced by the hydraulic pressure in the system. The net effect is a temporarily stucking of the control surface at its current position (Goupil et al., 2014). Although this phenomenon can be interpreted as a certain type of LOE fault, in most cases it has merely the character of a nuisance (thus to be ignored), due to its short time appearance. Nevertheless, since stall loads manifest typically at large control amplitudes, their fast detection and identification is important to distinguish them from other (e.g., additive) faults. Without explicitly recognizing stall loads, the detection of many additive faults in the case of large control inputs becomes more difficult due to the need for using larger detection thresholds which limit the smallest amplitude of detectable faults.
There are several ways to deal with the problem of a stall load. One is to use high fidelity models which include stall load effects as a basis for residual generator synthesis as presented by Ossmann (2014a). This requires the use of complex nonlinear models of the underlying actuator dynamics (e.g., the reference model) as part of the residual generator. However, to reduce the necessary computational efforts on the flight computers, much simpler models are to be preferred. In this paper a different approach is proposed which is based on simpler actuator models (thus simpler residual generators), by using a dedicated detection and identification strategy of stall load situations. Timely detection and identification of the onset and termination of stall loads allow a substantial enhancement of the aircraft situational awareness with potentially improved overall FDD system performance.

Note that the paper does not address the aircraft stall problem, but the actuator stall load phenomenon, which may occur at large deflections of control surfaces due to excessive aerodynamic loads acting on the actuator. In contrast, during a stall of the aircraft the actuator loads may decrease as the flow over the surfaces is separated.

The structure of the paper is as follows. In Section 2 an overview of the model-based synthesis methodology is presented. The architectures of two dedicated FDD system architectures are described in Sections 2.1 and 2.2 for the detection, isolation and identification of the parametric type of LOE fault as well as the stall load phenomenon. The development of a suitable LPV-model for the actuator dynamics as a basis for residual filter design is presented in Section 3 In Sections 4 and 5 detailed discussions of the setup of the two FDD systems are provided. In Section 6 the proposed detection and identification strategies are applied to an aircraft aileron actuator implemented in a nonlinear aircraft model. Examples and validation results of the detection and identification of the parametric type of LOE faults as well as of the stall load are presented.

\section{Model-based FDD}

Model-based fault diagnosis relies on suitable dynamical models of the system to be monitored. Typical components/blocks of any FDD system include (i) the residual generator to produce residual signals which indicate the presence or absence of a specific fault, (ii) the residual evaluation block to evaluate approximations of the residual signal norms, (iii) the decision making block to produce information on the presence or absence of a fault, (iv) an optional signal processing-based fault identification block to gather further information about the fault (e.g., fault classification).

In what follows, we discuss shortly two possible FDD system architectures suitable for monitoring parametric LOE faults and the stall load. 


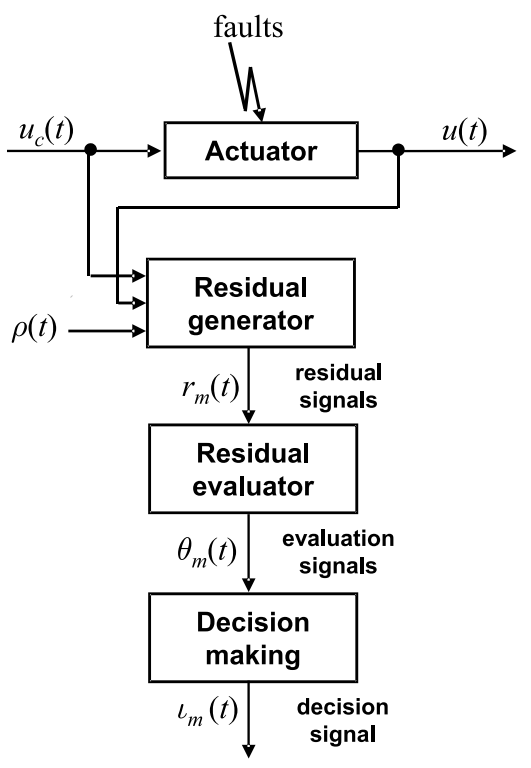

Fig. 1. FDD system for parametric LOE monitoring.

2.1. FDD system based on model detection. An FDD system architecture suited for monitoring parametric faults of flight actuators is presented in Fig. 1 and it is based on model-detection techniques such as those proposed by Varga (2009). The parametric faults are modeled as a collection of $N+1$ models, which includes the fault-free model and $N$ models describing relevant fault cases. The residual generator is formed of a bank of $N+1$ model detection filters, which process the commanded actuator position $u_{c}$ as well as the measured current actuator position $u$ and generate the corresponding components of the $(N+1)$-dimensional residual vector $r_{m}$. The $i$-th model detection filter generates the $i$-th component of $r_{m}$, which is a non-zero signal of significant magnitude for all measurement pairs $\left(u_{c}, u\right)$, except those which correspond to the $i$-th model, in which case the $i$-th component of $r_{m}$ will (ideally) be nearly zero. To cope with model uncertainties, robust model detection techniques are envisaged to be used based on LPV gain-scheduling techniques. For this purpose, the vector $\rho$ of scheduling variables is used as described in Section 3 . The residual evaluation block computes specific approximations $\theta_{m}$ of the norms of the components of the residual vector. These values are used in the decision making block, where a threshold-based decision logic is used to set the components of the signature vector $\iota_{m}$ to $0 / 1$ values, which codes the model detection results as follows: the $i$-th fault is detected provided all components of $\iota_{m}$ are 1 except for the $i$-th component, which must be 0 . Since the fault identification is implicitly performed by this detection schemes, no additional fault identification block is necessary.
2.2. FDD system for fault detection and identification. For the detection and identification of stall loads an FDD system with a traditional architecture as depicted in Fig. 2 can be used. Besides a dedicated robust (LPV-gain scheduled) residual generator with a scalar output $r$, the FDD system includes blocks for residual evaluation, decision making and stall load identification. In the residual evaluation block an approximation $\theta$ of the norm of the residual $r$ is computed. A threshold-based decision logic is employed to generate the decision signal $\iota$, which, if nonzero, triggers a specific signal based identification processes. The output of this identification block is the classification signal $\eta$, which indicates the presence $(\eta=$ $1)$ or absence $(\eta=0)$ of a stall load. The identification of the stall load consists in the determination of its main characteristics from the measured actuator input signal $u_{c}$ and the output signal $u$.

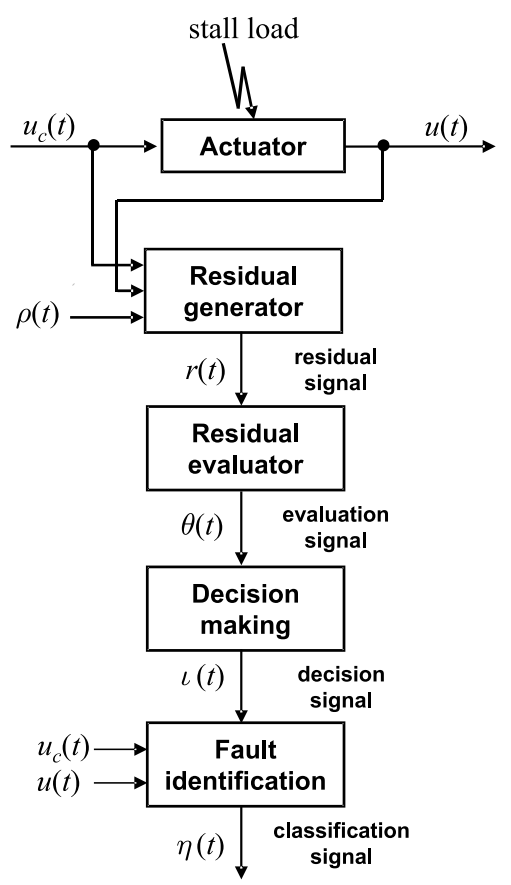

Fig. 2. FDD system for stall load detection and identification.

\section{Generation of LPV models}

Synthesis methodologies of FDD systems for various flight actuator failures must comply with strong requirements, by providing FDD-system designs which guarantee performance robustness (e.g., the lack of false alarms, the lack of missed detections, fast detection times) in all operating conditions and in the presence of model and environmental uncertainties. A prerequisite for model-based approaches for robust fault diagnosis is the availability of high-fidelity models to describe the 
complex actuator dynamics and their interaction with aerodynamics loads acting on the control surfaces. In this paper we rely on low complexity linear-parameter varying (LPV) actuator models to serve for synthesis purposes of FDD systems. The usefulness of such models in addressing robust diagnosis of various additive types of faults has been already demonstrated by Varga and Ossmann (2014).

In the case of parametric actuator faults, such as LOE types of faults, suitable parametric fault models are necessary to describe not only the fault-free case but also all relevant fault cases. In what follows, we apply the LPV-model generation approach of Varga et al. (2011) to generate LPV models starting from a high fidelity nonlinear actuator model which describes the two main categories of LOE faults. The resulting LPV models will serve FDD-system synthesis purposes.

Let $\nu=\left[\nu_{p, 1}, \nu_{p, 2}\right]$ be a two-dimensional vector whose first component $0<\nu_{p, 1} \leq 1$ is used to describe the effects of leakage (e.g., by lowering the available pressure), while the second component $0 \leq \nu_{p, 2} \leq$ 1 describes the effect surface damage or disconnection (e.g., by lowering the effect of aerodynamic force). The fault-free case corresponds to $\nu=[1,1]$. For the actuator with LOE faults we use a parameterized nonlinear dynamic model of a hydraulic servo controlled actuator described by a first-order nonlinear state equation (e.g., Goupil, 2010; Marton and Ossmann, 2012) of the form

$$
\dot{x}=K(p, x, \dot{x}, \nu)\left(u_{c}-x\right),
$$

with the output $u=x$, where $x$ and $u$ are the rod position, $u_{c}$ is the commanded position and

$$
\begin{aligned}
& K(p, x, \dot{x}, \nu) \\
& :=K_{c i} K_{p}\left[\frac { 1 } { \Delta P _ { \text { ref } } } \left(\nu_{p, 1} \Delta P(x)\right.\right. \\
& \left.\left.\quad-\left(\nu_{p, 1} \operatorname{sign}(\dot{x}) F_{\text {aero }}(p, x)+K_{d} \dot{x}^{2}\right) S^{-1}\right)\right]^{\frac{1}{2}},
\end{aligned}
$$

where $K_{p}$ is the servo control gain, $K_{c i}$ is a gain to convert an estimated current to a corresponding rod speed, $\Delta P$ is the hydraulic pressure delivered to the actuator, $\Delta P_{\text {ref }}$ is a differential pressure for a fully opened servo-valve (maximum rod speed), $F_{\text {aero }}$ represents the aerodynamic forces at the control surface, $K_{d} \dot{x}^{2}$ represents, in the case of an active/passive actuator scheme (Goupil, 2010), the estimated servo-control load of the adjacent actuator in damping mode, and $S$ is the actuator piston surface area. The components of the vector $p$ are flight parameters like the calibrated airspeed $V_{\text {cas }}$ and the aircraft altitude $h$. Observe that the state-space model (1) is already in a quasi-LPV form. However, the complex dependence of the gain $K(p, x, \dot{x}, \nu)$ in (2) on its arguments (e.g.,
$F_{\text {aero }}(p, x)$ is available only in a tabular form) limits the use of this model as basis for on-board implementations.

Let $\nu^{(i)}, i=0,1, \ldots, N$, be a collection of $N+1$ representative values of fault parameter $\nu$, which includes the fault-free case $\nu^{(0)}=[1,1]$. For each value $\nu=\nu^{(i)}$, we aim to obtain a simple LPV model

$$
\dot{x}=k^{(i)}(\rho)\left(u_{c}-x\right),
$$

which approximates (1) with good accuracy, where $\rho=(x, \operatorname{sign}(\dot{x}), p)$ is the vector of measurable parameters. The method employed by Varga et al. (2011) approximates the nonlinear gain $K(p, x, \dot{x}, \nu)$ by (2) by an easily computable gain $k\left(p, x, \dot{x}, \nu^{(i)}\right)$, which is then used in the first order actuator model as given in (3) as $k^{(i)}(\rho):=k\left(p, x, \dot{x}, \nu^{(i)}\right)$. Taking into account that the main variations of $K(p, x, \dot{x}, \nu)$ are caused by the aerodynamic force $F_{\text {aero }}(p, x)$ which acts on the control surface, the approximate gain is computed in the form

$$
\begin{aligned}
k(p, x, & \left.\dot{x}, \nu^{(i)}\right) \\
= & C_{0}\left(p, \nu^{(i)}\right)+C_{1}\left(p, \nu^{(i)}\right) \operatorname{sign}(\dot{x}) \\
& +x\left(C_{2}\left(p, \nu^{(i)}\right)+C_{3}\left(p, \nu^{(i)}\right) \operatorname{sign}(\dot{x})\right),
\end{aligned}
$$

where, for fixed $p, C_{0}\left(p, \nu^{(i)}\right)$ can be interpreted as the nominal gain, $C_{2}\left(p, \nu^{(i)}\right)$ describes the influence of the deflection angle $x$ on $k$, while the factor $\operatorname{sign}(\dot{x})$ allows distinguishing between upward and downward movements of the control surface, modeled by $C_{1}\left(p, \nu^{(i)}\right)$ and $C_{3}\left(p, \nu^{(i)}\right)$.

The chosen functional dependence on $x$ and $\operatorname{sign}(\dot{x})$ reflects the actual behavior of the actuator dynamics for different control surface positions and signs of the deflection rate. For $C_{i}\left(p, \nu^{(i)}\right), i=0,1,2,3$, affine approximations were used, where the intervening constant coefficients were determined using parameter fitting techniques based on comparing the output responses of the nonlinear actuator model (1) and the LPV-model (3). The final form of $k^{(i)}(\rho)$ is simple enough to be used in LPV-model based fault diagnosis applications.

\section{FDD system for detection and identification of parametric LOE faults}

Actuator faults due to leakage in the hydraulic system, or a broken actuator rod, or surface damage lead to changes in the actuator gain (2) and thus can be interpreted as a parametric type of LOE faults. Due to the lack of the strong fault detectability property, fault sensitivity is provided only during transients, and therefore the detection of such actuator faults using methods for additive faults is often difficult. Also, the solution of the associated fault identification problem (e.g., by using parameter estimation techniques) is challenging due to the 
time-varying nature of the actuator gain. In this section we describe an alternative approach to simultaneously achieve fault detection and fault identification using an approach based on model detection techniques. In what follows we discuss the setup of the main components of the FDD system in Fig. 1.

4.1. Residual generator for model detection. Consider $N+1$ LPV models of the actuator having the simple form (3),

$$
\begin{aligned}
& \dot{x}^{(i)}=k^{(i)}\left(\rho^{(i)}\right)\left(u_{c}-x^{(i)}\right), \\
& u^{(i)}=x^{(i)},
\end{aligned}
$$

which describe the relevant degradations of actuator performance (i.e., slower dynamics due to different degrees of the LOE type of faults) corresponding to the values $\nu^{(i)}$ for $i=0,1, \ldots, N$. Here

$$
\rho^{(i)}=\left(x^{(i)}, \operatorname{sign}\left(\dot{x}^{(i)}\right), p\right)
$$

is the vector of measurable parameters to be used for gain-scheduling.

To simplify the formal manipulations, we assume that each $\rho^{(i)}$ is constant, so we can alternatively use input-output representations of the form

$$
\mathbf{u}^{(i)}(s)=G^{(i)}\left(s, \rho^{(i)}\right) \mathbf{u}_{c}(s)
$$

for $i=0,1, \ldots, N$, with the transfer functions

$$
G^{(i)}\left(s, \rho^{(i)}\right)=\frac{k^{(i)}\left(\rho^{(i)}\right)}{s+k^{(i)}\left(\rho^{(i)}\right)} .
$$

$\mathbf{u}^{(i)}(s)$ and $\mathbf{u}_{c}(s)$ are the Laplace-transformed quantities of $u^{(i)}(t)$ and $u_{c}(t)$, respectively. The model detection problem can be formulated as follows (Varga, 2009): Determine a bank of $N+1$ filters with scalar outputs of the form

$$
\mathbf{r}^{(i)}(s)=Q^{(i)}\left(s, \rho^{(i)}\right)\left[\begin{array}{c}
\mathbf{u}(s) \\
\mathbf{u}_{c}(s)
\end{array}\right]
$$

with $i=0,1, \ldots, N$ and $\mathbf{u}(s)$ as the Laplace-transformed quantity of $u(t)$, such that for all $u_{c}(t)$ we have

(i) $r^{(i)}(t) \approx 0$ when $u(t)=u^{(i)}(t)$;

(ii) $r^{(i)}(t) \neq 0$ when $u(t)=u^{(j)}(t)$ for $i \neq j$;

(iii) $r^{(i)}(t)$ is asymptotically bounded.

We can fulfill the condition (i) by simply choosing

$$
Q^{(i)}\left(s, \rho^{(i)}\right)=M^{(i)}(s)\left[\begin{array}{ll}
1 & -G^{(i)}\left(s, \rho^{(i)}\right)
\end{array}\right],
$$

which is by construction stable if $M^{(i)}(s)$ is stable, so the condition (iii) is automatically fulfilled. $M^{(i)}(s)$ can be chosen to modify the dynamics of the filter. For example,
$M^{(i)}(s)=\left(G^{(i)}\left(s, \rho^{(i)}\right)\right)^{-1} G_{p}(s)$ ensures the same poles for all filters, defined by $G_{p}(s)$. Furthermore, we have, for $u(t)=u^{(j)}(t)$ and for $j \neq i$,

$$
\begin{aligned}
& \mathbf{r}^{(i)}(s) \\
& =Q^{(i)}\left(s, \rho^{(i)}\right)\left[\begin{array}{c}
\mathbf{u}^{(j)}(s) \\
\mathbf{u}_{c}(s)
\end{array}\right] \\
& =M^{(i)}(s)\left(G^{(j)}\left(s, \rho^{(j)}\right)-G^{(i)}\left(s, \rho^{(i)}\right)\right) \mathbf{u}_{c}(s),
\end{aligned}
$$

which shows that the condition (ii) is also fulfilled, provided $u_{c}(t)$ is nonzero. Due to the presence of a zero in the origin of $G^{(j)}\left(s, \rho^{(j)}\right)-G^{(i)}\left(s, \rho^{(i)}\right)$, strong fault detectability is not provided. Thus, for successful model detection, $u_{c}(t)$ must not be a step signal.

The overall residual generator used for model detection results in the form

$\mathbf{r}_{m}(s):=\left[\begin{array}{c}\mathbf{r}^{(0)}(s) \\ \mathbf{r}^{(1)}(s) \\ \vdots \\ \mathbf{r}^{(N)}(s)\end{array}\right]=\left[\begin{array}{c}Q^{(0)}\left(s, \rho^{(0)}\right) \\ Q^{(1)}\left(s, \rho^{(1)}\right) \\ \vdots \\ Q^{(N)}\left(s, \rho^{(N)}\right)\end{array}\right]\left[\begin{array}{c}\mathbf{u}(s) \\ \mathbf{u}_{c}(s)\end{array}\right]$

4.2. Residual evaluation and decision making. For the evaluation of the magnitude of the residual signal $r^{(i)}(t)$ for $i=0, \ldots, N$, we employ the so-called Narendra signal evaluation scheme of the form (Narendra and Balakrishnan, 1997)

$$
\begin{aligned}
\theta^{(i)}(t)= & \alpha^{(i)}\left|r^{(i)}(t)\right| \\
& +\beta^{(i)} \int_{0}^{t} e^{-\gamma^{(i)}(t-\tau)}\left|r^{(i)}(\tau)\right| \mathrm{d} \tau
\end{aligned}
$$

where $\theta^{(i)}(t)$ can be generated by the first order filter

$$
\begin{aligned}
& \dot{\xi}^{(i)}(t)=-\gamma^{(i)} \xi^{(i)}(t)+\beta^{(i)}\left|r^{(i)}(t)\right|, \\
& \theta^{(i)}(t)=\xi^{(i)}(t)+\alpha^{(i)}\left|r^{(i)}(t)\right| .
\end{aligned}
$$

The filter parameters $\alpha^{(i)} \geq 0$ and $\beta^{(i)} \geq 0$ are suitable weights for instantaneous and long-term values, respectively, while $\gamma^{(i)}>0$ is the forgetting factor and can be set to different values for different models.

For the detection of the $i$-th model, all components of the residual vector $r_{m}(t)$ must be nonzero, except for the $i$-th component. For each component $r^{(i)}(t)$ of the $N+1$-dimensional residual vector $r_{m}(t)$ an evaluation signal $\theta^{(i)}(t)$ of the form (13) can be generated to build the evaluation vector

$$
\theta_{m}(t):=\left[\theta^{(0)}(t), \ldots, \theta^{(N)}(t)\right]^{T},
$$

and define the decision vector $\iota_{m}$ as

$$
\iota_{m}(t):=\left[\iota^{(0)}(t), \ldots, \iota^{(N)}(t)\right]^{T},
$$


whose $i$-th component is defined as

$$
\iota^{(i)}(t)= \begin{cases}1, & \text { if } \theta^{(i)} \geq \tau^{(i)}\|u\|_{2} \\ 0 & \text { otherwise }\end{cases}
$$

where $\tau^{(i)}$ is a suitable threshold for nonzero gains. For the threshold selection we can exploit (11) and choose

$$
\tau^{(i)}:=\inf _{\substack{j \neq i \\ \rho^{(j)}, \rho^{(i)}}}\left\|G^{(j)}\left(s, \rho^{(j)}\right)-G^{(i)}\left(s, \rho^{(i)}\right)\right\|_{\infty} .
$$

Furthermore, with suitable scalings of the filters (14), it is possible to use a unique value, say $\tau^{(i)}=\tau_{m}$, for all thresholds.

Regarding decision making, note that the only valid signatures in $\iota_{m}$ for model detection are those with a single zero component. Therefore, all other signatures, such as, e.g., all components nonzero or all components zero, rule out cases when there is no LOE type of fault or the detection conditions $u_{c} \neq 0$ or $u_{c}$ nonconstant are not fulfilled. Note that this implicitly includes the fact, that the parametric LOE actuator fault cannot be detected with the proposed FDD system if the input is zero.

\section{FDD system for detection and identification of stall loads}

In this section we describe the detection and identification of the stall load phenomenon, which occurs when the sum of antagonist forces to which the actuator has to act exceeds the available hydraulic pressure in the system. In this intermittent situation the control surface seems to be temporarily stuck at its current position. This load from a faulty behavior is to increase the detection threshold so that the residual induced by a stall load does not trigger an alarm. Another possibility is to delay possible detections, as the time of occurrence of a stall load is limited. Both approaches result in a degradation of the detection performance of the FDD system (e.g., detection times, minimum detectable fault magnitudes). However, this is unacceptable in some cases.

These circumstances provide the motivation to develop a dedicated detection and identification procedure for stall loads. If the stall load situation is clearly identified, the need for decreased thresholds or delayed detections disappears and with it the degradation of the resulting FDD system performance. Due to the intermittent characteristics of stall loads, the required situation awareness and hence the detection and identification have to be achieved in a minimum of time. Therefore, optimization based techniques will be used to provide the best detection times together with a maximum of robustness.

5.1. LPV residual generator for detection of stall loads. Assume temporarily that the parameters in $\rho$ are constant. In this case we can use an input-output representation of the actuator fault model in the form,

$$
\mathbf{u}(s)=G(s, \rho)\left(\mathbf{u}_{c}(s)+\mathbf{f}(s)\right),
$$

where $\mathbf{u}(s), \mathbf{u}_{c}(s)$, and $\mathbf{f}(s)$ are the Laplace-transformed quantities of $u(t), u_{c}(t)$, and the fault $f(t)$, respectively. In (17), $G(s, \rho)$ is a parameter dependent transfer functions corresponding to (3)

$$
G(s, \rho)=\frac{k(\rho)}{s+k(\rho)} .
$$

As a residual generator we use a parameter dependent filter of the form

$$
\mathbf{r}(s)=Q(s, \rho)\left[\begin{array}{c}
\mathbf{u}(s) \\
\mathbf{u}_{c}(s)
\end{array}\right],
$$

where $Q(s, \rho)$ is the $1 \times 2$ transfer-function matrix of the filter, which explicitly depends on the measurable parameter $\rho$ (e.g., via an equivalent state-space realization of the filter). For a physically realizable filter, $Q(s, \rho)$ must be robustly stable for all values of $\rho$. The robust fault detection synthesis problem addresses the robustness of the fault detection system with respect to the measurable parameter $\rho$ by attempting to achieve robustness using an LPV gain scheduling approach.

To address robust detection of the LOE, we employed the synthesis method described by Varga et al. (2011). Accordingly, we can use a first order detector of the form

$$
Q(s, \rho)=\left[\begin{array}{ll}
\frac{a}{k_{0}} \frac{s+k(\rho)}{s+a} & -\frac{k(\rho) a}{k_{0}(s+a)}
\end{array}\right],
$$

where $a$ is an arbitrary positive value specifying the dynamics of the detector and $k_{0}$ is a typical nominal value of the gain $k(\rho)$. By replacing in 19) $\mathbf{u}(s)$ by its expression in 17), we obtain the internal form of the detector,

$$
\mathbf{r}(s)=R_{u_{c}}(s, \rho) \mathbf{u}_{c}(s)+R_{f}(s, \rho) \mathbf{f}(s),
$$

where

$$
\begin{aligned}
& {\left[R_{u_{c}}(s, \rho) \mid R_{f}(s, \rho)\right]} \\
& \quad:=Q(s, \rho)\left[\begin{array}{c|c}
G(s, \rho) & G(s, \rho) \\
1 & 0
\end{array}\right] .
\end{aligned}
$$

The choice 20) of $Q(s, \rho)$ guarantees an exact decoupling of control inputs in (22). Thus $R_{u_{c}}(s, \rho)=0$. The corresponding fault-to-residual transfer function is

$$
R_{f}(s, \rho)=\frac{k(\rho)}{k_{0}} \frac{a}{s+a} .
$$

The LPV state-space realization of the residual generator (19) can be always obtained in the form

$$
\begin{gathered}
\dot{x}_{Q}(t)=A_{Q} x_{Q}(t)+B_{Q}(\rho)\left[\begin{array}{c}
u(t) \\
u_{c}(t)
\end{array}\right], \\
r(t)=C_{Q} x_{Q}(t)+D_{Q}(\rho)\left[\begin{array}{c}
u(t) \\
u_{c}(t)
\end{array}\right] .
\end{gathered}
$$


For the detector (20), the state-space matrices are

$$
\begin{aligned}
A_{Q} & =-a, & B_{Q}(\rho) & =a\left[\begin{array}{ll}
\frac{k(\rho)-a}{k_{0}} & \frac{k(\rho)}{k_{0}}
\end{array}\right], \\
C_{Q} & =1, & D_{Q} & =\left[\begin{array}{ll}
\frac{a}{k_{0}} & 0
\end{array}\right] .
\end{aligned}
$$

The chosen form 20 of the detection filter leads to a state-space realization with a constant feed-through matrix $D_{Q}$. This has the major advantage of preventing all direct effects on $r(t)$ of the discontinuities in the scheduling signal $\rho$ (e.g., jumps due to the presence of the signum-function in (4)).

5.2. Residual evaluation and decision making. For the evaluation of the scalar residual, the Narendra signal evaluation scheme (14) is used. In this case, the evaluation signal $\theta(t)$ is compared to a specific threshold $\tau$ in the decision making process to determine the decision signal $\iota(t)$ using the decision logic

$$
\iota(t)= \begin{cases}1 & \text { if } \theta(t) \geq \tau \\ 0 & \text { otherwise }\end{cases}
$$

The signal $\theta(t)$ is ideally equal to zero or sufficiently small in fault free situations, whereas it will exceed the threshold $\tau$ when a stall load occurs. Hence, for the detection of the stall load the appropriate selection of the values of the free parameters $\alpha, \beta$ or $\gamma$, together with an appropriate threshold $\tau$, essentially influences the performance of the FDD system.

5.3. Determination of the detection threshold. The free parameters $\alpha, \beta$ and $\gamma$ of the residual evaluation blocks and the threshold $\tau$ used in the decision blocks must be chosen to ensure that the requirements regarding typical performance criteria used in the industry such as the false alarm rate (FAR), the missed detection rate (MDR) or the detection time performance (DTP) are fulfilled. Simultaneous minimization of these quantities (e.g., by using multi-objective optimization techniques) would provide the best achievable detection performance. Unfortunately, analytical expressions of the FAR and the MDR are not available, and only surrogates can be used, which are suitable only when we require FAR $=0$ and $\mathrm{MDR}=0$. The applicability of such surrogates based on tuning has been demonstrated by Varga et al. (2011).

For an optimization based tuning setup, the requirements for the lack of false alarms and missed detections can be formulated as either optimization criteria or constraints. In the absence of faults, the requirement for no false alarms leads to a constraint on the false alarm bound,

$$
\tau_{f}:=\sup _{f=0} \theta(t)<\tau,
$$

where the supremum is taken for all admissible operation points, all relevant aircraft maneuvers, all admissible variations of uncertain parameters and all relevant disturbances.

The requirement for no missed detection can be also expressed as a constraint on the detection bound,

$$
\tau_{d}:=\inf _{t \in\left[t_{f}, t_{\mathrm{detec}}\right]} \theta(t) \geq \tau,
$$

which must be satisfied for all relevant stall load situations. Here, $t_{\text {detec }}$ is the maximum admissible detection time and $t_{f}$ is the stall load occurrence time. To ensure simultaneously the lack of false alarms and of missed detections, the condition $\tau_{f}<\tau_{d}$ must be fulfilled. Various optimization strategies are presented by Varga and Ossmann (2014).

A positive detection gap $\tau_{d}-\tau_{f}$ can be interpreted as a robustness measure of the fault detection performance. Note that the worst-case parameter combinations resulting from the computation of $\tau_{f}$ in 26) and $\tau_{d}$ in (27) are usually different. If $\tau_{d}-\tau_{f}>0$ holds, a constant threshold $\tau$ satisfying $\tau_{d} \leq \tau<\tau_{f}$ can be chosen to guarantee no false alarms and no missed detections. A choice of $\tau$ near to $\tau_{f}$ allows in general shorter detection times.

For the computation of $\tau_{f}$ and $\tau_{d}$, solving global worst-case optimization problems to find the worst-case parameter combinations appears to be the most adequate choice. However, less demanding computational approaches can be used, such as a gridding based worst-case search over the flight envelope and parameter space, or Monte-Carlo simulations, to determine approximations of the upper bound $\tau_{f}$ and lower bound $\tau_{d}$.

5.4. Stall load identification. Due to its time limited occurrence, fast identification of the stall load is the main requirement for the design of the identification logic. Two different approaches to identify the stall load will be presented in what follows. The first, more complex one is based on model detection methods, similar to the detection and identification approach presented for the parametric type of LOE fault. The second one is based on a signal based analysis, showing reduced complexity but increased robustness.

5.4.1. Identification via model detection. To identify the stall load using a model detection approach, a dedicated model of the actuator dynamics during the stall load needs to be generated. If a stall load occurs, nearly no movement of the actuator rod is possible. Thus, a linear first order approximation with the actuator gain (1) simplifying to a constant gain $k(\rho)=k_{s l}$ can be used. Typical values for the gain $k_{s l}$ lie between 0.01 and 0.1 . 
To be able to address the identification of stall loads via model detection, a residual filter in the form

$$
Q_{s l}(s)=M(s)\left[1-\frac{k_{s l}}{s+k_{s l}}\right]
$$

is proposed, where $M(s)$ can be selected to modify the filter dynamics. For example, with the inverse of the actuator dynamics and $a_{s l}$ being an arbitrary positive value, $M(s)$ can be specified as

$$
M(s)=\frac{s+k_{s l}}{k_{s l}} \frac{a_{s l}}{s+a_{s l}},
$$

resulting in the filter

$$
Q_{s l}(s)=\left[\begin{array}{ll}
\frac{a_{s l}}{k_{s l}} \frac{s+k_{s l}}{s+a_{s l}} & -\frac{a_{s l}}{s+a_{s l}}
\end{array}\right] .
$$

The output of the residual filter

$$
\mathbf{r}_{s l}(s)=Q_{s l}(s)\left[\begin{array}{c}
\mathbf{u}(s) \\
\mathbf{u}_{c}(s)
\end{array}\right]
$$

will be zero or sufficiently small if the modeled dynamics correspond to the behavior of the real actuator, while and nonzero otherwise. To improve the robustness of the detection, the output of the residual filter is evaluated with a Narendra filter (14) before comparing it to a dedicated threshold $\tau_{s l}$. Note that a residual value below this threshold does not directly indicate a stall load. The residual will be lower than $\tau_{s l}$ if no input is present $\left(u_{c}=\right.$ 0 ) or very slow movements of the actuator are requested by the input signal $u_{c}$. Hence, only the combination of a sufficiently large residual $r$ of the residual of filter (19) and a sufficiently small residual $r_{s l}(t)$ leads to the identification of a stall load situation.

5.4.2. Signal-based identification. Signal-based identification approaches offer an easier but robust way to identify stall load situations. As described by Goupil et al. (2014), during a stall load the surface seems to be stuck at its current position. Hence, the signal based identification logic for jammed actuators presented by Varga and Ossmann (2014) using a variance measure of the output signal provides a good starting point for signal based stall load identification. However, the main drawback with regard to fast identification times of this approach is the variance computation, which requires a set of collected values to be collected. As for the stall load the identification has to be very fast; the required variation of the actuator output is approximated via its mean value at time $t_{i}$ by

$$
\overline{\dot{u}} \approx \frac{u\left(t_{i}\right)-u\left(t_{d}\right)}{t_{i}-t_{d}},
$$

where $t_{d}$ is the detection time and $t_{i}=t_{d}+i T$, $i=1,2, \ldots$, with $T$ being a suitable sampling period.
Early identification of the stall load can then be done by checking that the actuator deflection variation rate is below or above a certain slew rate $\dot{u}_{\text {min }}$ respectively,

$$
|\bar{u}| \leq \dot{u}_{\min }
$$

where $\overline{\dot{u}}$ is the mean value of the derivative over a given time period.

As all other relevant actuator faults, except for jamming, in general show a nonzero variance on the output signal, the stall load can be discriminated from these faults using the condition (33). While the detection and identification of jamming faults is primary interesting at low deflection signals (Varga et al., 2013), stall loads usually occur at extensive input maneuvers where the aerodynamic forces acting on the control surface are high. This fact can be used to discriminate between a jamming fault and a stall load. Let $\tau_{u_{c, \max }}$ and $\tau_{u_{\max }}$ be the threshold for large input and output signals, respectively, which can be used to discriminate between normal (care-free) operations and operations with large inputs and outputs, where potentially jamming is of more interest:

$$
\begin{aligned}
\left|u_{c}\right| & \geq \tau_{u_{c \max }}, \\
|u| & \geq \tau_{u_{\max }} .
\end{aligned}
$$

Finally, to add more robustness against other fault identification schemes, a stall load situation requires that the sign of the input and output signal be the same and the absolute value of the output signal be lower than the absolute value of the input signal. These two conditions can be summarized in a single one by

$$
\operatorname{sign}\left(u_{c}-u\right)=\operatorname{sign}(u) .
$$

To confirm a stall load, we set $\eta(t)=1$ if the three conditions (33), (34) and (35) are fulfilled.

Note that signal processing based identification of the stall load is only activated if the evaluated residual of the residual of the filter (19) is large enough to cross its threshold $\tau$, so that the output of the decision block is set to $\iota=1$. This separation between detection and identification is a distinctive feature of this approach, when compared with other techniques, such as those used by Goupil (2010) and Gheorghe et al. (2013), where fault identification algorithms must run permanently on on-board flight control computers (Varga and Ossmann, 2014).

\section{Application examples}

In this section we describe the application of the methodologies described in this paper to the detection and identification of parametric LOE faults and stall loads of an aileron surface controlled by a hydraulic actuator. The nonlinear actuator model of the aileron is part of 
a nonlinear model of a closed-loop aircraft including a nonlinear control law ensuring robust stability over the whole flight envelope.

6.1. Parametric LOE. Figure 3 shows the output signals of the fault-free actuator and of a faulty actuator with a loss of the hydraulic pressure by $25 \%$ occurring at $15 \mathrm{~s}$. As only the dynamical behavior of the actuator is influenced, there is only a small difference in the two signals, making the parametric LOE fault nearly undetectable using additive fault based approaches. This is the reason why the model detection based approach is used for the detection and identification of this type of LOE fault. For the case of a parametric LOE fault, we employed six actuator models for the following pairs of fault parameters: $\nu^{(0)}:=[1,1]$ for the fault free case, $\nu^{(1)}:=[1,0], \nu^{(2)}:=[1,0.33]$, and $\nu^{(3)}:=$ $[1,0.66]$, for a $100 \%, 66 \%$ and $33 \%$ loss of the control surface effectiveness, respectively, and $\nu^{(4)}:=[0.85,1]$ and $\nu^{(5)}:=[0.73,1]$, for a $15 \%$ and $27 \%$ loss of the hydraulic pressure, respectively. Correspondingly, approximations of the actuator gains $k^{(i)}(\rho)$ of the form (4) were determined using parameter fitting techniques described in Section 3. In the special case of a $100 \%$ LOE due to either actuator disconnection or total surface damage, no aerodynamic force is acting on the actuator dynamics any more. In this case, the gain (1) simplifies to the following easily implementable exact gain:

$$
k^{(1)}(\rho)=K_{c i} K_{p} \sqrt{\frac{\Delta P(x)}{\Delta P_{\text {ref }}}},
$$

which can be used alternatively.

6.1.1. Parameter setting. The six actuator models of the form (7) were used to define the corresponding six scalar output detectors (10), which enter in the overall detector (12). For all six Narendra filters in the evaluation block, the same values of the parameters were used, namely, $\alpha=0, \beta=1, \gamma=0.1$. Using suitable scaling of the individual detectors, the same detection threshold value of $\tau_{m}=0.15$ was used for all $\tau^{(i)}$.

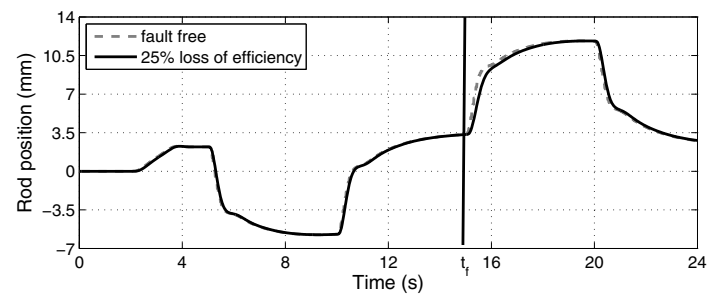

Fig. 3. Effect of the parametric LOE.

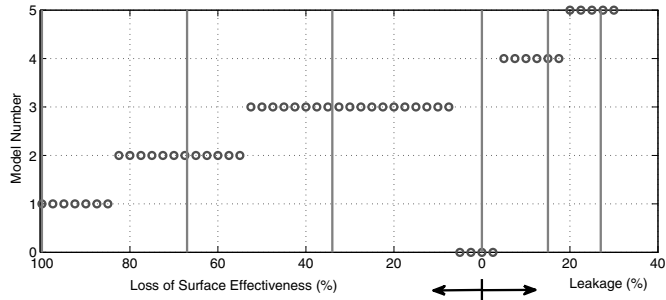

Fig. 4. Detected model during fault simulations.

6.1.2. Verification and validation results. Figure 4 shows the results of model detection for different degradations of the control surface between $0 \%$ and $100 \%$ as well as for leakage in the pressure supply of up to $30 \%$ on a dedicated point in the flight envelope. The vertical lines indicate the grid values corresponding to the faults for which an LPV model was developed and used in the detector. The circles show the results of the model identification obtained using the closed-loop flight control system augmented with the developed FDD system. Important to notice is that all models near a grid value were correctly detected (i.e., there are no switching between models), and therefore no false classification of the fault occurred. More accurate fault identification can be obtained by increasing the number of models (with the associated increased computational load).

The model detection approach for the parametric LOE was tested on different points in the flight envelope with different maneuvers to check the false alarm rate as well as the detection performance. No false alarms were encountered during the analysis. As the detection time and its demanded value highly depends on the size of the fault (i.e., the degree of efficiency degradation), it is reasonable to analyze the detection times for different fault sizes separately. As an example, the worst-case detection time in the relevant flight envelope for the complete loss of surface effectiveness was determined at approximately 10 $\mathrm{s}$, while the required detection time was $120 \mathrm{~s}$, indicating good detection time performance.

6.2. Stall load. Figure 5 shows a typical stall load situation. During a high input maneuver the aerodynamic force to which the actuator has to act temporarily exceeds the available hydraulic pressure. This results in two stall loads situations of up to $5 \mathrm{~s}$, where the actuator output $u$ is not able to follow its commanded input $u_{c}$, which can be seen in the first diagram of Fig. 5. The second diagram shows the normalized pressure difference $\Delta P-\operatorname{sign}(\dot{x}) F_{\text {aero }} / S$, which is the available pressure used to move the actuator. This pressure difference is dropping to zero at around $4 \mathrm{~s}$ and $16 \mathrm{~s}$ simulation time, resulting in stall load situations. In the third diagram the residual signal of the detector 20 is depicted, clearly indicating 

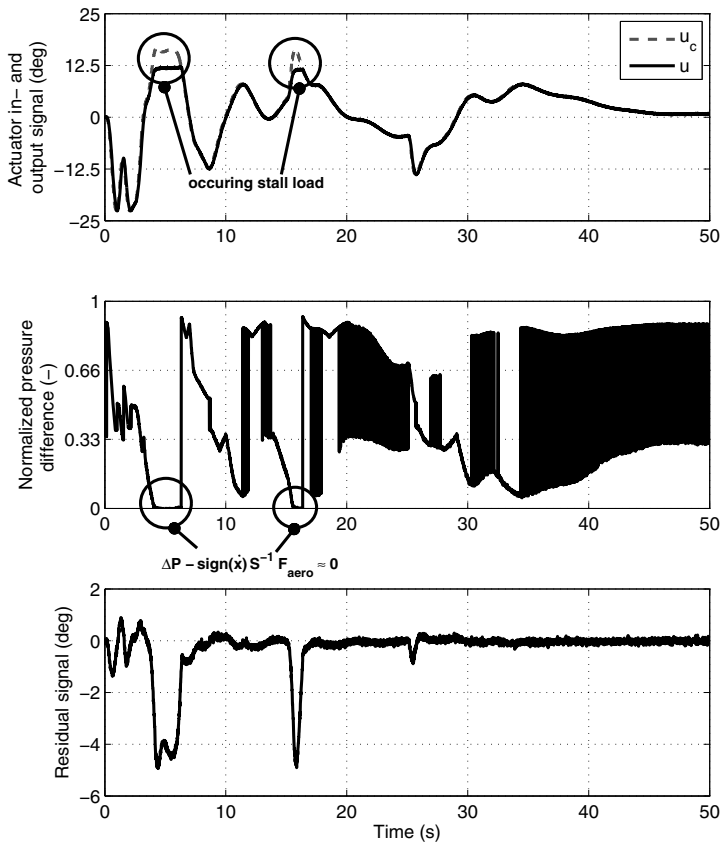

Fig. 5. Stall loads during a high input maneuver.

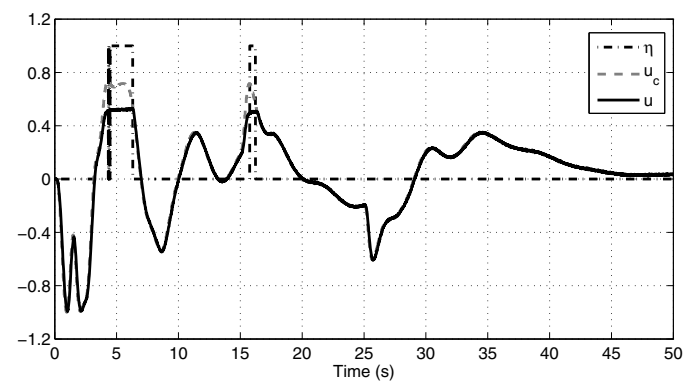

Fig. 6. Stall load identification signal.

an increased residual caused by the stall load situations.

Without dedicated identification of these situations the thresholds in an FDD system for monitoring actuator faults need to be increased or the detection and identification of faults has to be delayed so that the stall loads do not lead to any false alarm. Applying the presented detection and identification strategy for stall loads in Section 5.4 these steps and the resulting degradation of FDD system performance can be avoided. In Fig. 6 the stall load identification signal $\eta$ is depicted beside the normalized input and output of the actuator. This clearly indicates fast detection and identification of the stall load situations. With this awareness, the thresholds of other fault detection and identification channels (see Varga and Ossmann, 2014) can be maintained low.

6.2.1. Parameter tuning. The free parameters of the fault evaluation and decision block can be determined in an optimal way, such that typical requirements as a lack of false alarms and missed detections, besides constraints on the detection time, which is the most important aspect for the detection of a stall load, are fulfilled. Multi-objective optimization based tuning strategies has been described, for example, by Ossmann (2014b) as well as Ossmann and Varga (2013). For the tuning of the FDD system, the values of the parameters $\alpha, \beta$ and $\gamma$ were determined to guarantee a fast detection time and fulfill a positive detection gap $\tau_{d}-\tau_{f}$. The parameters of the Narendra filter are set to $\alpha=1, \beta=1, \gamma=0.1$, while the threshold is set to $\tau=2.5$. The parameter values of the identification strategy presented in Section 5.4 were set using the relevant stall load signal characteristics. Recall that the signal processing based identification of the stall load is only triggered if the detection has been successful.

6.2.2. Verification and validation results. The robustness of the designed FDD system was thoroughly tested for both stall load free and stall load situations in the whole flight envelope and a full range of aircraft parameter variations. To check for the lack of false alarms, typical maneuvers such as, for example, piloted flights with various pilot inputs (longitudinal/lateral stick doublets, pedal input demand, nose up and nose down demands) or typical navigation maneuvers (level flight, flight path angle target mode, yaw angle target mode, speed change, steady sideslip, coordinated turn, etc.) were successfully used. Various high input maneuvers (lateral stick doublets, roll protection maneuvers, constant sideslip maneuvers) were simulated on different points in the flight envelope to check the detection and identification performance, providing satisfactory results. All relevant stall load situations were detected below half a second.

\section{Conclusions}

In this paper we proposed a model-based synthesis and tuning methodology for the development of an FDD system for the detection and identification of parametric loss of efficiency failure cases as well as stall load situations for aircraft actuators. The main features of the proposed approach, which confers superior performance over existing approaches, include (i) relying on accurate LPV models allowing the synthesis of robust fault detection filters, (ii) application of advanced synthesis methods of LPV residual generators guaranteeing robust fault detection, (iii) employing integrated optimization-based tuning of the parameters of the fault evaluation and decision making blocks, including the determination of detection thresholds to guarantee no false alarms and no missed detections, (iv) employing a real-time implementable fault identification method.

Using a dedicated identification strategy for stall load situations, the performance of FDD systems is no 
larger degraded by the need of higher threshold values or delayed detections. An important aspect to mention is that while the synthesis of LPV residual generators relies on a fault monitoring approach at the component (actuator) level, the tuning of the overall FDD system parameters and the final verification and validation involve the closed-loop aircraft. The proposed methodologies were successfully applied for the design of an FDD system for the identification of parametric LOE faults and stall loads of an aileron actuator.

An open question not addressed in this article is how the derived information about the fault situation can be used for reconfiguring the flight control system. This is the topic of ongoing research within the FP7 RECONFIG$U R E$ project.

\section{Acknowledgment}

This work was performed in the framework of the FP7 RECONFIGURE project (grant agreement no. AAT-2012-RTD-2314544).

\section{References}

Boškovic, J.D., Bergstrom, S.E. and Mehra, R.K. (2005). Adaptive accommodation of failures in second-order flight control actuators with measurable rates, American Control Conference, Portland, OR, USA, pp. 1033-1038.

Caglayan, A.K., Rahnamai, K. and Allen, S. (1988). Detection, identification and estimation of surface damage/actuator failure for high performance aircraft, American Control Conference, Atlanta, GA, USA, pp. 2206-2212.

Eykeren, L.V., Chu, Q. and Mulder, J. (2012). Actuator fault detection by aerodynamic model identification, 8th IFAC Symposium on Fault Detection, Supervision and Safety for Technical Processes, Mexico City, Mexico, pp. 1353-1357.

Gheorghe, A., Zolghadri, A., Cieslak, J., Goupil, P., Dayre, R. and Berre, H.L. (2013). Model-based approaches for fast and robust fault detection in an aircraft control surface servo loop, Control Systems Magazine 33(3): 20-30.

Goupil, P. (2010). Oscillatory failure case detection in the A380 electrical flight control system by analytical redundancy, Control Engineering Practice 18(9): 1110-1119.

Goupil, P., Boada-Bauxell, J., Marcos, A., Cortet, E., Kerr, M. and Costa, H. (2014). AIRBUS efforts towards advanced real-time fault diagnosis and fault tolerant control, 19th IFAC World Congress, Cape Town, South Africa, pp. 3471-3476.

Marton, L. and Ossmann, D. (2012). Energetic approach for control surface disconnection fault detection in hydraulic aircraft actuators, 8th IFAC Symposium on Fault Detection, Supervision and Safety for Technical Processes, Mexico City, Mexico, pp. 1149-1154.

Narendra, K.S. and Balakrishnan, J. (1997). Adaptive control using multiple models, IEEE Transactions on Automatic Control 42(2): 171-187.
Ossmann, D. (2014a). Fault Detection, Isolation and Identification in Electro-hydraulic Actuators of Modern Aircaft, Ph.D. thesis, Technical University of Munich, Munich.

Ossmann, D. (2014b). Optimization based tuning of fault detection and diagnosis systems for safety critical systems, 19th IFAC World Congress, Cape Town, South Africa, pp. 8570-8575.

Ossmann, D. and Varga, A. (2013). Progress in Flight Dynamics, Guidance, Navigation, Control, Fault Detection, and Avionics, Torus Press, Moscow, pp. 263-281.

Varga, A. (2009). Least order fault and model detection using multi-models, Conference on Decision and Control, Shanghai, China, pp. 1015-1019.

Varga, A., Hecker, S. and Ossmann, D. (2011). Diagnosis of actuator faults using LPV-gain scheduling techniques, AIAA Guidance, Navigation, and Control Conference, Portland, OR, USA

Varga, A. and Ossmann, D. (2014). LPV-techniques based robust diagnosis of flight actuator faults, Control Engineering Practice 31: 135-147.

Varga, A., Ossmann, D., Goupil, P. and Sabot, G. (2013). Verification and validation of a FDD system for identification of flight actuator jamming, 19th IFAC Symposium on Automatic Control in Aerospace, Wuerzburg, Germany, pp. 84-89.

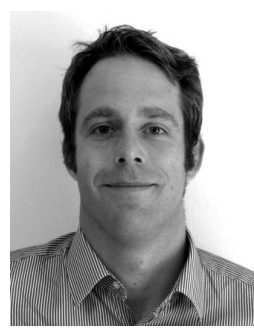

Daniel Ossmann received his first M.Sc. degree in aerospace engineering from the University of Applied Sciences in Graz in 2006. He received his second M.Sc. degree in 2008 and his Ph.D. degree in 2014, both in mechanical engineering from the Technical University of Munich. Since 2009 he has been a research associate at the Institute of System Dynamics and Control at the German Aerospace Center (DLR) in Oberpfaffenhofen. His main research interests lie in the field of fault detection and diagnosis, fault tolerant and robust control for aerospace applications as well as flying and handling qualities. So far he has published more than 20 papers in journals, books and conferences, and holds two patents.

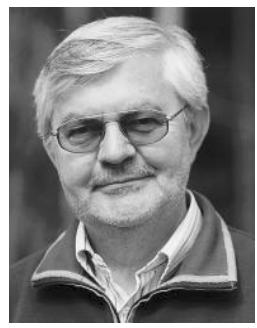

Andreas Varga is a senior scientist at the Institute of System Dynamics and Control of the German Aerospace Center (DLR) in Oberpfaffenhofen. He received a diploma in control engineering in 1974 and a Ph.D. degree in electrical engineering in 1981, both from the University "Politechnica" of Bucharest (Romania). His main research interests include numerical methods for linear systems analysis and design (with special emphasis on model and controller reduction, descriptor systems, periodic systems, fault detection), and robust numerical software for computer aided control system design. He has coauthored three books, coedited two books, published over 65 papers in refereed journals or book chapters, and has over 155 conference publications. Dr. Varga is a fellow of the IEEE and served as an associate editor for IEEE Transactions on Automatic Control in 1997-1999.

Received: 30 January 2014 Revised: 31 March 2014 